\title{
Gradhiva
}

GRADHIV

Revue d'anthropologie et d'histoire des arts

$8 \mid 2008$

Mémoire de l'esclavage au Bénin

\section{Mémoire de l'esclavage et capital religieux}

Les pérégrinations du culte egun dans la région d'Abomey

Memory of slavery and religious capital. The peregrinations of the Egun cult in the Abomey region

Joël Noret

\section{OpenEdition}

Journals

Édition électronique

URL : http://journals.openedition.org/gradhiva/1183

DOI : 10.4000/gradhiva. 1183

ISSN : $1760-849 x$

Éditeur

Musée du quai Branly Jacques Chirac

Édition imprimée

Date de publication : 15 novembre 2008

Pagination : 48-63

ISBN : 978-2-915133-94-3

ISSN : 0764-8928

\section{Référence électronique}

Joël Noret, « Mémoire de l'esclavage et capital religieux », Gradhiva [En ligne], 8 | 2008, mis en ligne le 15 novembre 2011, consulté le 01 mai 2019. URL : http://journals.openedition.org/gradhiva/1183 ; DOI : 10.4000/gradhiva. 1183 


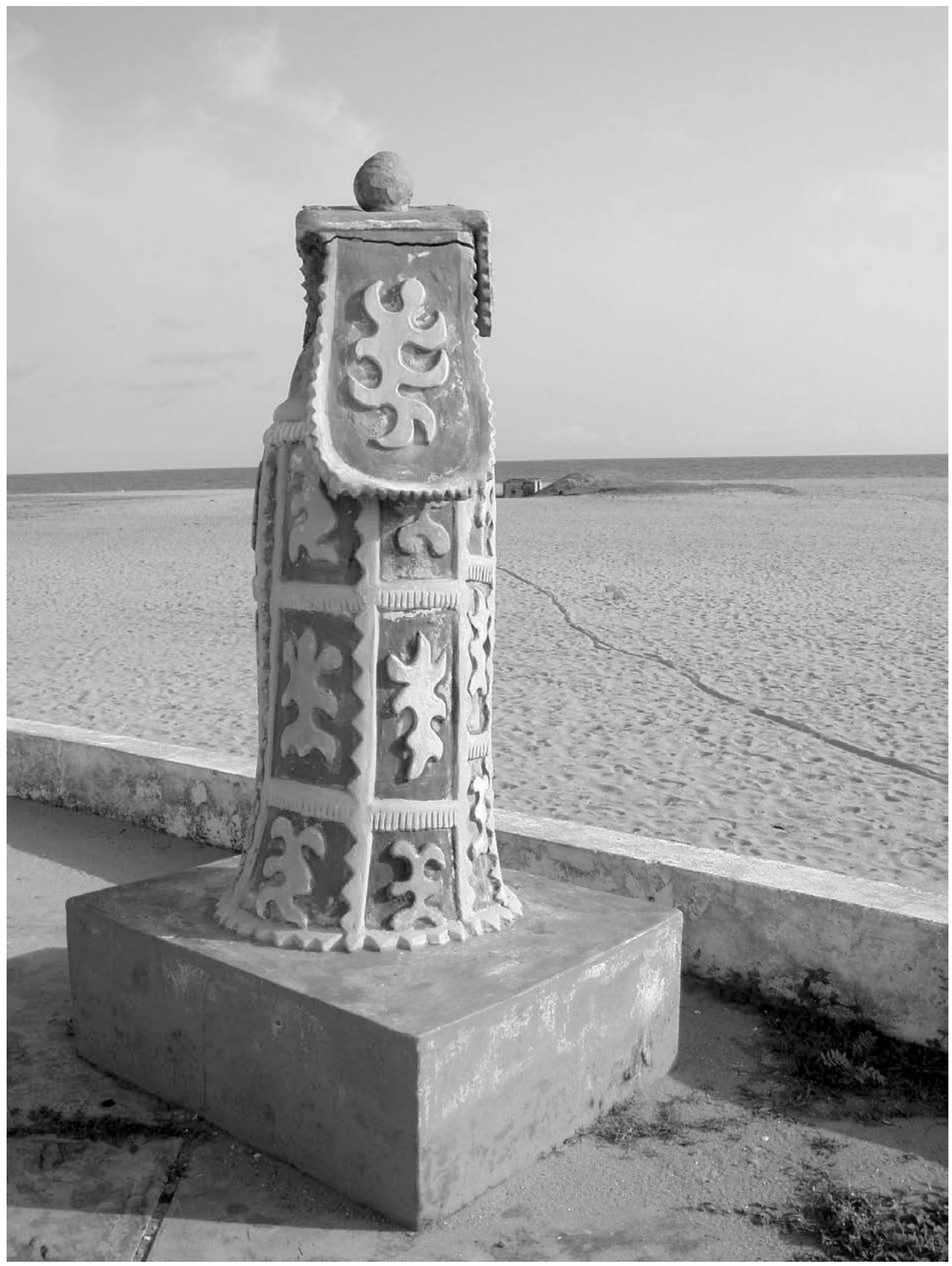

Fig. 1 Face à l'océan, de dos, l'une des deux statues d'egun qui encadrent la Porte du Non-Retour. Euvre d'Yves Kpède, 2005. Photo G. Ciarcia. 


\title{
Mémoire de l'esclavage et capital religieux
}

\author{
Les pérégrinations du culte egun dans la région d’Abomey
}

\section{Introduction}

L'anthropologue ou l'historien familier du Bénin méridional contemporain ne peut qu'être frappé par l'écart qui existe aujourd'hui, dans la région, entre d'une part l'inflation des commémorations de la traite transatlantique et d'autre part le silence ou la gêne qui continuent régulièrement à entourer la question de l'esclavage local, et ce alors que sur le territoire du royaume précolonial du Dahomey, région qui sera ici évoquée en particulier, cet esclavage fut pratiqué, faut-il le rappeler, par la grande majorité des lignages ${ }^{1}$.

Bien entendu, les rapports à la mémoire de l'esclavage local sont, dans une certaine mesure tout au moins, multiples, comme l'ont été les positions sociales à l'époque où ce système était en vigueur, et comme elles le sont aujourd'hui. Les descendants des maîtres de jadis ont facilement sur la chose des points de vue différents de ceux des descendants d'esclaves, d'affranchis ou de dépendants, lesquels euphémisent souvent bien davantage que les premiers leur condition d'alors dans les récits livrés à l'ethnographe. Toutefois, la position occupée par les lignages dans le système esclavagiste est loin de constituer le seul élément qui intervienne dans la formation de l'espace des points de vue sur l'esclavage local coexistant dans le Bénin méridional. Le niveau d'éducation, la réussite sociale et/ou économique, la socialisation religieuse, les rapports entretenus avec son lignage ou avec sa "grande famille ", comme on dit, en un mot la diversité des conditions d'existence et des trajectoires sociales, pèsent aussi de tout leur poids, cela va de soi, dans le rapport que les individus entretiennent avec le passé de l'esclavage. Sur ce point comme sur bien d'autres, il n'est donc pas raisonnable de prétendre livrer un seul " native point of view " : l'anthropologie n'a pas vocation à «imposer des modèles grossiers sur des gens subtils » (Clifford $1986: 9$ ).

Dans les pages qui suivent, je chercherai à éclairer certains aspects des mémoires de l'esclavage local ${ }^{2}$ à travers les regards portés aujourd'hui dans la région d'Abomey, au cœur du pays fon, sur une forme yoruba de culte aux ancêtres. Ce culte est celui des egun ou des « revenants ", comme on dit dans le français local, et ses cérémonies culminent avec la sortie (contrôlée par une société initiatique) de masques d'ancêtres. Depuis vingt ans au moins, il est progressivement devenu, dans la

1. Cet article repose principalement sur plusieurs mois d'enquêtes menées à Abomey entre 2004 et 2007, avec la collaboration de Constant Legonou, sur l'histoire des familles et des cultes aux ancêtres yoruba dans la région. D'autres enquêtes ont été réalisées pendant la même période sur l'histoire de ces cultes à Ouidah, et sont ici mobilisées de façon plus ponctuelle. Enfin, cet article a été rédigé pendant une année passée comme Visiting Fellow à l'université d'Oxford avec le soutien de la fondation Wiener-Anspach (Université libre de Bruxelles); David Berliner, Gaetano Ciarcia et Jacques Noret ont bien voulu relire une version antérieure de ce texte : qu'ils trouvent ici l'expression renouvelée de mes remerciements.

2. Je renvoie ici, à travers la notion de mémoire, dont on sait la prolifération dans l'anthropologie contemporaine (voir Berliner 2005), aux seules mémoires discursives de l'esclavage. Ma problématique est donc, on l'aura compris, quelque peu différente de celle de certains travaux récents qui cherchent à explorer les «mémoires pratiques 》 (Shaw 2002) ou les marques laissées par l'esclavage sur une série d'institutions religieuses dans certaines sociétés africaines impliquées dans la traite transatlantique des esclaves (voir aussi Argenti 2006; Argenti et Röschenthaler 2006). 


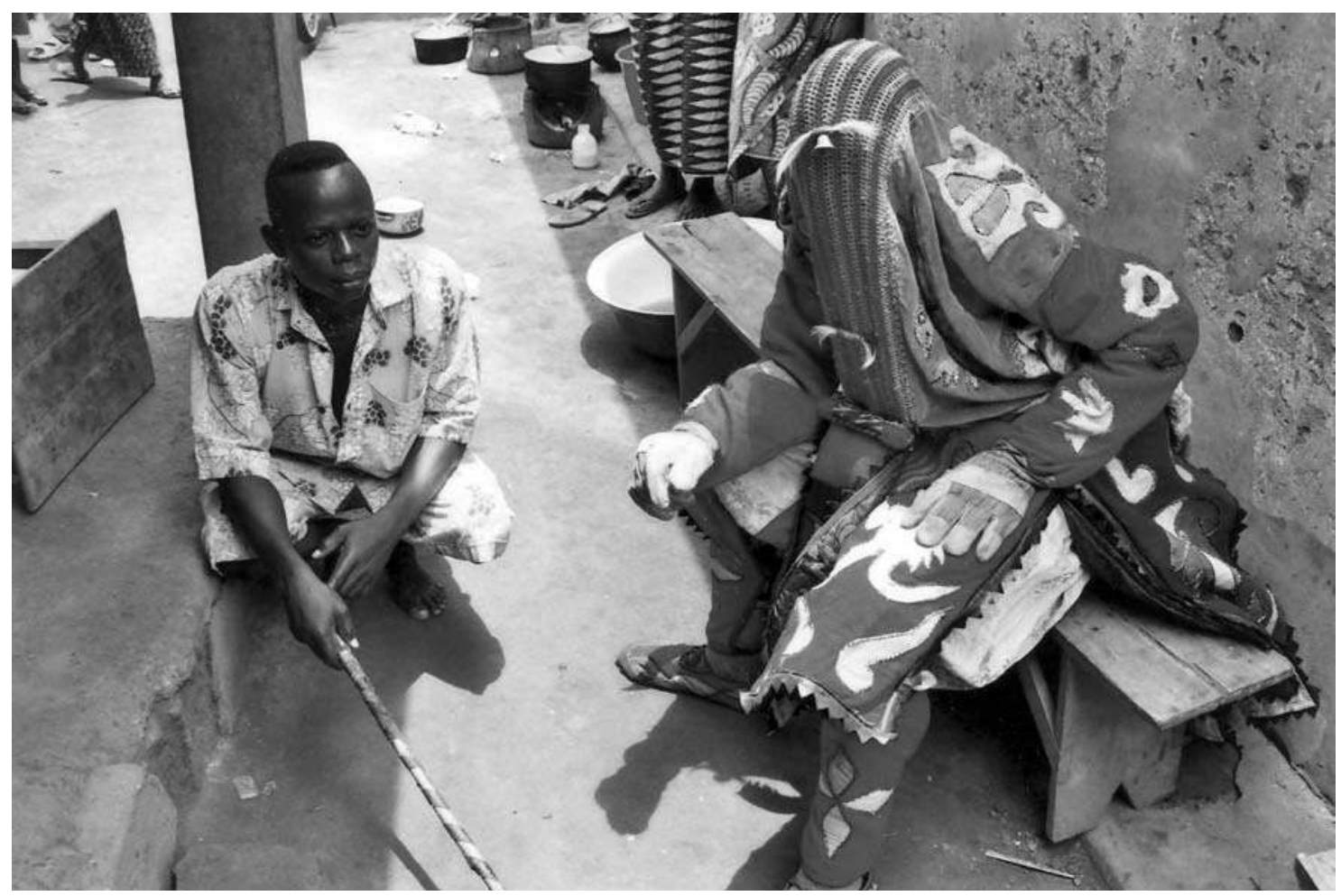

Fig. 2 Un egun et son guide assis dans la cour d'une concession lignagère fon à Abomey, 2005. Photo J. Noret.

région d'Abomey, au cœur de l'ancien royaume fon du Dahomey, un emblème de l'identité yoruba, laquelle renvoie ici presque toujours à une ascendance marquée par l'expérience honteuse de l'esclavage, ou tout au moins de la dépendance ou de l'assujettissement ${ }^{3}$.

\section{Le culte egun à Abomey en contexte : du stigmate au chevron}

À la différence d'autres cultes, d'origine yoruba ou autre, que les Fon acquirent suite à des victoires militaires ou à des alliances avec des groupes voisins (selon la dynamique, bien connue dans cette partie de l'Afrique au moins, de cumul des puissances et d'appropriation des forces religieuses d'autrui; voir Augé 1982), le culte egun ne fut pas encouragé à Abomey (ni d'ailleurs à Ouidah, le port du royaume où vécurent aussi bon nombre d'esclaves et d'affranchis yoruba, voir Law 2004 : 95 ; Noret 2006 : 466-477). Est-ce parce que, comme le culte oro, lui aussi souvent associé aux ancêtres dans certaines régions du pays yoruba, et lui aussi initialement découragé par les Fon, le culte des egun repose sur une société initiatique masculine? Cet élément a probablement joué4. On sait que le royaume du Dahomey s'est montré fort méfiant envers les sociétés initiati- ques (il semble ainsi ne pas y avoir eu, dans le Dahomey précolonial, de société de masques, voir Herskovits 1938a : 242-249; Law 2004 : 95), et même envers les cultes vodun, dont les chefs pouvaient toujours être suspectés de fomenter un complot politique dans leurs « couvents " réservés aux initiés (voir Le Hérissé 1911 : 132-133;

3. Le royaume fon du Dahomey fut, au XIX ${ }^{\mathrm{e}}$ siècle, la principale formation politique de l'actuel Bénin méridional. II contrôlait toute la partie centrale de celui-ci, depuis les villages au nord d'Abomey, la capitale, jusqu'à la région de Ouidah, le port à travers lequel se faisait le commerce avec diverses puissances européennes. La bande nord-sud ainsi contrôlée en permanence par le royaume avait une largeur d'une quarantaine ou une cinquantaine de kilomètres (entre la rivière Couffo à l'ouest, et la rivière Zou puis le fleuve Ouémé à l'est ), mais les incursions et les razzias des troupes aboméennes menaçaient aussi chaque année une zone de plusieurs dizaines de kilomètres à l'est et au nord de la capitale, les expéditions des troupes du Dahomey ayant mené celles-ci au nord de Dassa et de Savalou (au nord), ou jusqu'à Kétou, Pobè et au-delà (à l'est)

4. Un certain nombre d'éléments présentés ici valent non seulement pour les difficultés rencontrées par le culte egun dans la région d'Abomey, mais aussi pour l'histoire du culte oro, autre forme de culte aux ancêtres pratiquée à l'origine par certains groupes (en particulier les Ègba) de l'ensemble de populations qu'on a commencé à désigner comme yoruba au XIX ${ }^{e}$ siècle (voir sur ce dernier point Peel 2000). Comme le culte egun, oro est contrôlé par une société initiatique masculine seule autorisée à fréquenter les espaces d'où les oro font entendre leur voix inarticulée, tantôt ronflante, tantôt stridente (voir Noret 2006 : 340-366, 461-489). 
Herskovits 1938a : 242-244, 1938b : 175-178). Mais un autre aspect du système esclavagiste local a probablement joué un rôle tout aussi important dans la répression du culte egun par le pouvoir royal : en milieu urbain et dans les lignages fon au moins, les esclaves étaient progressivement intégrés à des positions subalternes dans le lignage de leurs maîtres, se voyant notamment imposer les rites de naissance et de mort de ceux-ci5. Laisser aux esclaves, qu'ils soient ou non d'origine yoruba ${ }^{6}$, la possibilité d'organiser le culte de leurs propres ancêtres posait évidemment un problème majeur dans le cadre d'un tel modèle assimilationniste.

Un autre point toutefois doit encore être souligné. La force du modèle assimilationniste fon n'était pas la même selon le statut et les compétences des esclaves : on sait bien aujourd'hui que le couple maître-esclave peut se révéler trop simpliste pour rendre compte des diverses formes de dépendance et d'assujettissement qui se sont mises en place dans les sociétés africaines précoloniales (sur le cas, proche, du pays yoruba, voir notamment Peel 2000 : 63-64). Au Dahomey, les escla-

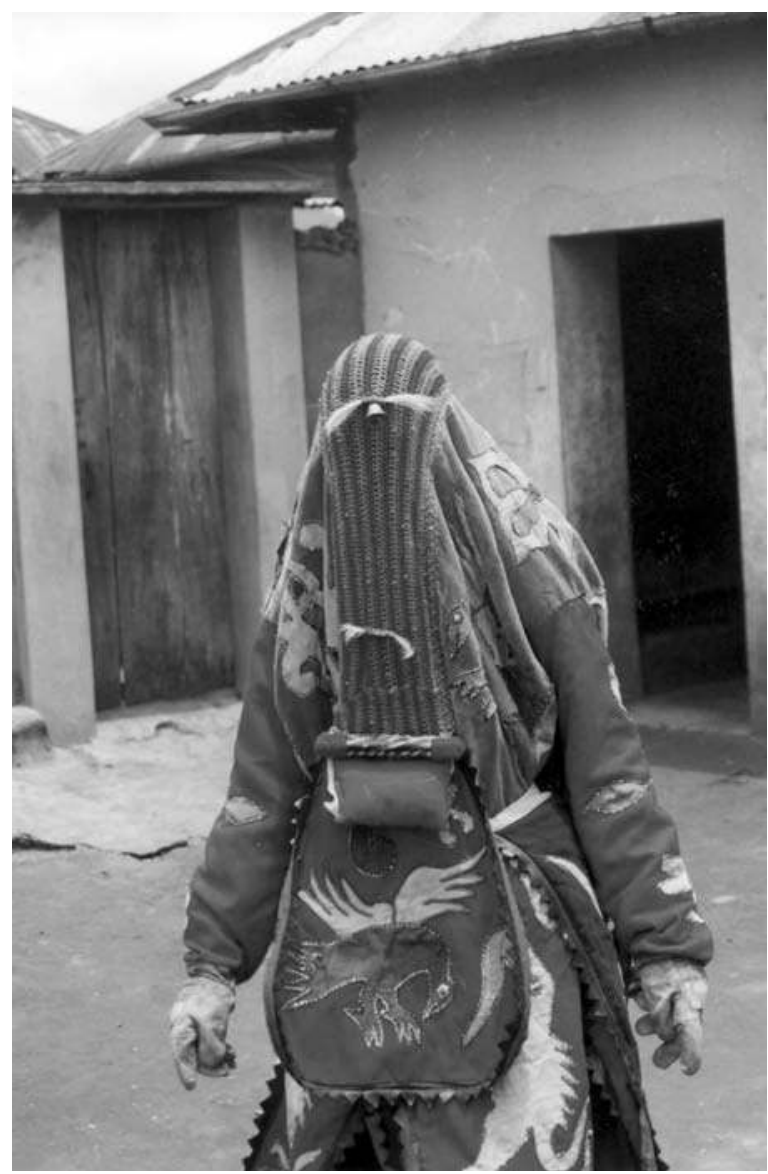

Fig. 3 Un egun dans la cour d'une concession lignagère fon à Abomey, 2005. Photo J. Noret. ves, yoruba en particulier, qui se révélaient posséder des compétences de devin pouvaient ainsi accéder assez rapidement à une relative indépendance, voire à une reconnaissance sociale importante. Le cas le plus éloquent de ce type de trajectoire est probablement celui de Guèdègbe, le devin des rois Glèlè (1858-1888) puis Béhanzin (1889-1894), rendu célèbre par le travail de Bernard Maupoil (1943).

Fils d'une femme capturée à Savè dont une des filles devint une épouse du roi Glèlè (1858-1888), né à Abomey, Guèdègbe se vit confier, une fois ses compétences religieuses reconnues, la responsabilité de tous les cultes aux oricha (divinités) yoruba présents à Abomey. Ni oro ni egun ne semblent cependant avoir fait partie de ceuxci. Jusque récemment, il n'y avait d'ailleurs pas d'autels de ces cultes dans la concession lignagère. Dans la famille Guèdègbe, les funérailles et le culte des ancêtres prirent d'abord une forme largement fon : à l'issue du cycle des rites funéraires fon, c'est un assin, un autel d'ancêtre fon, qui était installé pour les membres défunts du lignage en formation. Toutefois, des séquences de rites funéraires yoruba, signes d'une mémoire en miettes (voir Bastide 1970 : 95-108; Mary 2000 : 39-43) de la tradition lignagère antérieure à l'arrivée à Abomey, restaient présentes dans ce schéma, comme l'installation d'une figuration provisoire yoruba du défunt parallèlement aux rites d'ancestralisation fon. Cependant, à l'issue du processus, le seul autel d'ancêtre installé était fon?

\footnotetext{
5. La situation était la même dans les lignages d'autres populations soumises par les Fon à date plus ancienne, et devenues alliées, comme les Hweda de Ouidah. Les lignages hweda qui ont acquis des esclaves yoruba à la fin du XVIII et au XIX ${ }^{e}$ siècle ont eu eux aussi tendance à les intégrer progressivement à des positions subalternes dans leurs lignages. Dans les familles afro-brésiliennes de Ouidah, en revanche (qui s'installèrent dans la ville à partir du premier tiers du XIX ${ }^{\mathrm{e}}$ siècle), si certains esclaves se sont progressivement vu attribuer (et ont pu revendiquer) le nom de leurs maîtres, il n'y avait pas de rites lignagers qui pouvaient être imposés aux esclaves, comme ce fut le cas dans bon nombre de lignages fon. Et il semble, selon les traditions orales de certaines familles yoruba, que certains esclaves ou dépendants de familles afrobrésiliennes ont pu organiser leur propre culte aux ancêtres, au moins sous une forme minimale (n'impliquant probablement pas, dans le cas des familles yoruba adeptes d'egun, la sortie de masques), dès la fin du xIx siècle (voir Noret 2006 : 466-479).
}

6. Si cet article porte en priorité sur l'articulation entre la mémoire de l'esclavage et un culte aux ancêtres yoruba, il y a eu, dans le royaume du Dahomey, des esclaves et des dépendants de bien d'autres origines. Les Mahi, par exemple, nom donné par les Fon aux populations immédiatement voisines du Dahomey au nord et à l'est (voir Anignikin 2001), furent également nombreux à être capturés et réduits en esclavage par les troupes aboméennes.

7. Entretiens réguliers avec sranon Guèdègbe (août 2005, août 2006 et août 2007), ajaho Guèdègbe (idem) et Élisabeth Guèdègbe (août 2007). 
En fait, c'est seulement aux alentours de 1920 que le culte egun semble avoir fait son apparition à Abomey ${ }^{8}$ (Noret 2006 : 482-485). En 1938, Melville Herskovits, le premier à en signaler l'existence ${ }^{9}$, écrit, après son séjour à Abomey en 1931, que «les chefs [fon], bien qu'ils n'y soient pas résignés, se préparent à le reconnaître comme un phénomène à prendre en considération, dans la mesure où les groupes egun deviennent importants et nombreux" (1938a : 245). Herskovits témoigne ainsi d'une affirmation progressive mais indéniable des lignages yoruba en formation dans l'espace public, et décrit notamment une sortie de masques egun à proximité immédiate du marché central d'Abomey, le marché étant dans cette région, on le sait, le cœur de la ville et de la vie sociale.

Cette émergence de cultes lignagers yoruba apparaît comme manifestement conflictuelle. L'un des accompagnateurs d'Herskovits lors de cette sortie de masques, issu d'un lignage noble fon (il s'agit probablement de J. Aho ou de l'un de ses proches, voir Preston Blier 1989), commente la performance des egun avec une certaine agressivité : " "Pourquoi devrions-nous les avoir ici [les egun], alors que nous avons nos propres tovodun?" " (Herskovits 1938a : 246). Une intervention qu'Herskovits commente à son tour de façon pertinente : «La menace exercée par cette société [initiatique] sur le culte dahoméen des ancêtres [auquel il est fait allusion à travers le culte des tovodun] et à travers celui-ci sur le contrôle [social] exercé par le lignage royal peut être considérée, à partir d'une telle remarque, comme quelque chose que réalise clairement la caste régnante dahoméenne " (ibid.). C'est peu dire donc que « les sujets coloniaux [...] ont vu le passé depuis des perspectives différentes et variées " (Lambek et Antze 1996 : xiv).

En fait, le premier masque egun à être sorti à Abomey fut, selon les sources orales les plus autorisées, un masque nommé Onduboye, qui fut amené vers 1920 par des gens de Ouidah, ville moins étroitement contrôlée par les Fon (une bonne partie des esclaves et dépendants yoruba y avaient par exemple des maîtres afro-brésiliens), où le culte egun avait émergé plus rapidement (Noret 2006 : 466-479), probablement dès la dernière décennie du $\mathrm{XIX}^{\mathrm{e}}$ ou les premières années du $\mathrm{xx}^{\mathrm{e}}$ siècle $^{10}$. La première sortie d'Onduboye semble avoir agi à Abomey comme un véritable déclencheur, et les premiers revenants appartenant à des familles yoruba de la ville sortirent ensuite rapidement dans les deux zones de l'agglomération où avaient été regroupées les concessions des esclaves yoruba, à savoir les quartiers Hountondji et Azali. Il est difficile de savoir aujourd'hui quelles furent effectivement les premières familles à faire sortir des masques, car la primauté en ce domaine est un enjeu de légitimité important, les sources écrites manquent et les récits entendus dans différentes familles sont loin de toujours concorder : chacun tend à donner à sa lignée le premier rôle. Il est clair cependant que, aux débuts du culte, celui-ci ne concerne pas l'ensemble des Yoruba (le devin Guèdègbe, par exemple, avait interdit, dans les premières décennies du $x^{\mathrm{e}}$ siècle, que des egun se produisent dans sa maison ou devant celle-ci), mais seulement certaines familles, qui se savent elegun, c'est-à-dire " adeptes d'egun".

Assez rapidement toutefois, la conjonction de plusieurs facteurs produisit un contexte favorable à une reconnaissance sociale plus importante du culte, et à son appropriation par un nombre croissant de lignages : tout d'abord, la perte de pertinence au quotidien, sous la colonisation, de la distinction maître-esclave, conjuguée à l'apparition et au développement de nouveaux critères de différenciation et de hiérarchie sociales (liés à l'école ou à l'adoption du christianisme, notamment) ; ensuite l'attrait d'une société initiatique comme celle du culte egun pour bien des hommes (en quête de connaissances ésotériques nouvelles et/ou de titres de notabilité procurés par telle ou telle charge en son sein); et enfin les décès de femmes yoruba toujours

8. Je dépends essentiellement, pour les éléments de l'histoire du culte egun à Abomey évoqués ci-dessous, d'entretiens réguliers menés entre août 2004 et août 2007, à Abomey et à Cotonou, avec baba Azagoumangba, balè Sagbadjou (aujourd'hui décédé), alagba B. Bocokpè, balè Akankossi, baa Nondicawo et Paul Zodji.

9. Aucune mention d'egun ni d'oro n'est, par exemple, faite par Auguste Le Hérissé dans l'ouvrage qu'il consacre en 1911 à L'Ancien Royaume du Dahomey (Le Hérissé 1911)

10. Signalons ici les affirmations peu étayées d'Edna Bay sur la chronologie de l'apparition des cultes egun et oro sur le territoire de l'ancien royaume du Dahomey. Celle-ci soutient en effet que «la présence des sociétés secrètes Oro et Egungun est rapportée par les Aboméens au règne de Guezo [1818-1858]. Egungun, qui pourrait avoir été la première société de masques à avoir été autorisée au Dahomey, fut rebaptisée Kutito [...]. Même des familles afro-brésiliennes de premier plan à Ouidah ont connu une transformation de leur culture luso-catholique à cette époque, adoptant Kutito et Oro, ainsi que de nombreuses [autres] divinités yoruba, grâce à des femmes amenées dans les familles comme femmes-esclaves » (Bay 1998: 190 et 257). Edna Bay n'étaie cependant ces affirmations rapides sur l'importation précoce des sociétés initiatiques egun et oro à Abomey que par un seul entretien, mené à Ouidah en 1972 avec un homme qui l'a vraisemblablement induite en erreur. En outre, s'il ne faut pas sous-estimer le rôle des épouses yoruba dans les lignages fon, notamment dans l'acquisition par ceux-ci, après la mort de ces femmes, des cultes egun et oro, ce phénomène n'est intervenu que plus tard, au $x x^{\mathrm{e}}$ siècle. Enfin, Edna Bay semble tout récemment être revenue sur ses affirmations sur le développement précoce du culte egun dans le royaume du Dahomey, écrivant maintenant dans son dernier ouvrage que les sociétés de masques y «étaient interdites » (Bay $2008: 142$ ). 
plus nombreuses dans les lignages fon (et donc la liaison par les femmes d'un nombre croissant d'individus aux Yoruba). L'appartenance au monde des initiés d'egun (et d'oro) devint ainsi progressivement au fil du xx siècle, et dans les dernières décennies en particulier, un marqueur important de l'identité masculine dans les milieux populaires aboméens, où l'affiliation à une Église reste faible et concerne surtout les femmes. La quête, puis la revendication du statut d'initié sont aujourd'hui bien souvent constitutives de l'affirmation de leur masculinité pour les jeunes hommes de la ville ${ }^{11}$.

Le droit à l'installation d'un autel egun est donc peu à peu revendiqué par un nombre toujours plus important de lignages, qu'il s'agisse de lignages yoruba manifestant l'intention d'organiser ce qui leur semble être le véritable culte de leurs ancêtres, ou de lignages fon souhaitant faire une place à la tradition ancestrale supposée des épouses yoruba du lignage, jusque-là inhumées selon les rites du lignage de leur mari. En d'autres termes, le prestige peu à peu associé au culte attire à celui-ci à la fois des Yoruba dont les liens historiques avec le culte ne sont pas toujours évidents (on sait l'hétérogénéité interne de ce qui est devenu à partir du
XIX ${ }^{\mathrm{e}}$ siècle, et à la suite d'une sorte d'invention missionnaire, le monde yoruba, voir Peel 2000 ; Mary 2003) et des Fon qui revendiquent désormais la place occupée dans leur lignage par des épouses d'une quelconque origine yoruba pour se poser eux aussi en détenteurs du culte.

En outre, parallèlement à son émergence, le culte est évidemment diabolisé, comme les autres cultes « traditionnels ", par les missionnaires et les Églises chrétiennes (dont la scène est largement dominée par l'Église catholique dans la région d'Abomey). La possession d'un autel egun et les connaissances s'y rapportant deviennent néanmoins un capital religieux légitime, tandis que le culte devient à différents égards un emblème de l'identité yoruba. Quoique fort populaire aujourd'hui, le culte des egun est aussi, désormais, accompagné d'un discours sur la perte de puissance des masques et le pouvoir supérieur qu'ils auraient autrefois détenu (« avant, ce que les masques disaient [les bénédictions],

11. Cet état de fait concerne aussi, au moins, la région de Ouidah, comme en témoignent les commentaires qui me furent adressés par plusieurs amis après mon initiation dans le culte egun : «Tu es un homme, maintenant! »

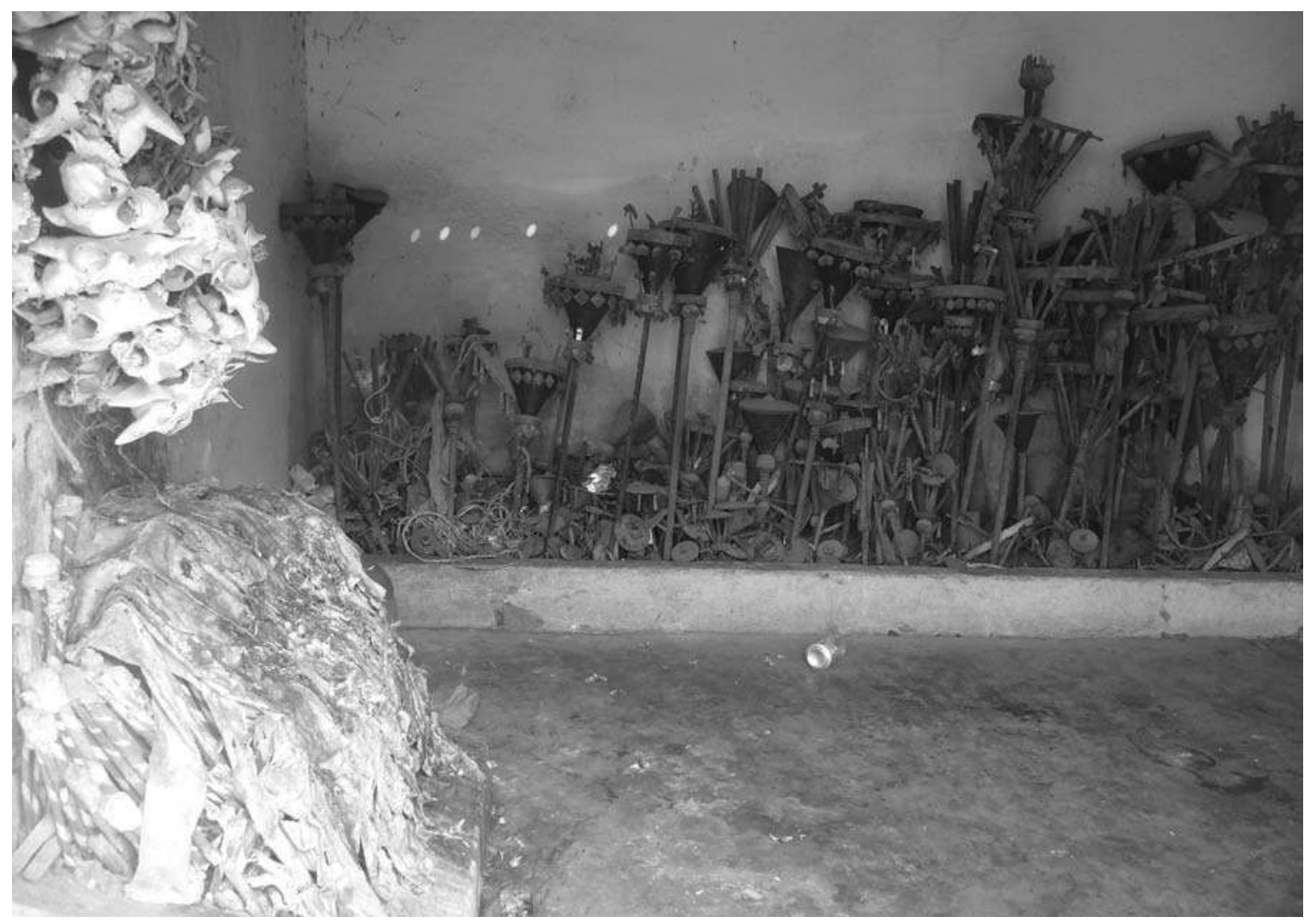

Fig. 4 À l'avant-plan à gauche, sous une grappe de crânes de cabris, l'actuel autel d'ancêtres yoruba de la famille Guèdègbe. À l'arrière-plan, les autels ancestraux fon qui servaient à la famille à honorer ses morts jusqu'à il y a une vingtaine d'années. Abomey, août 2007. Photo J. Noret. 


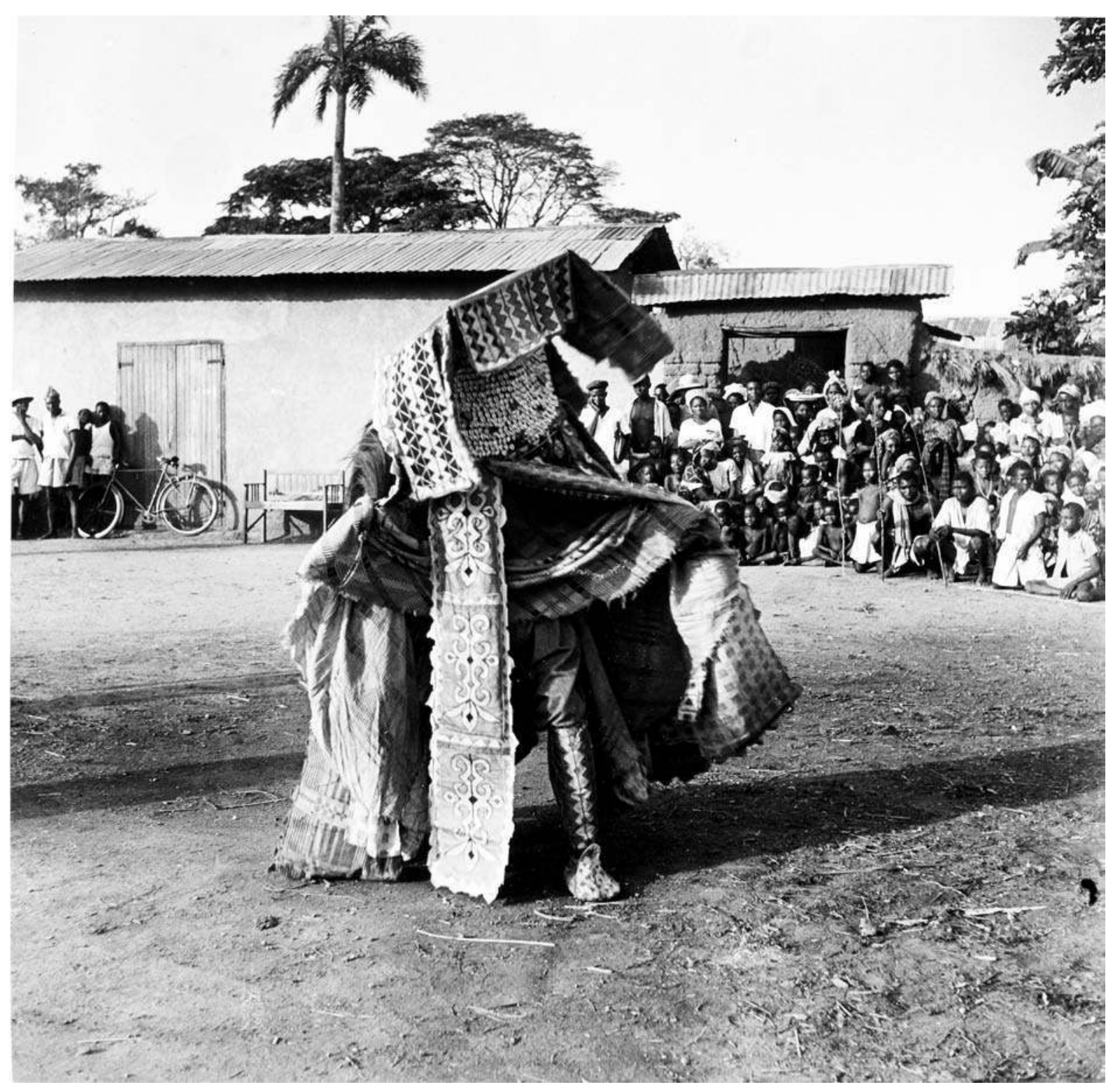

Fig. 5 Pierre Verger, « Pagne d’Egoun en Afrique, Ouidah, Dahomey », 1949-1953 @ musée du quai Branly/fondation Pierre Verger. 


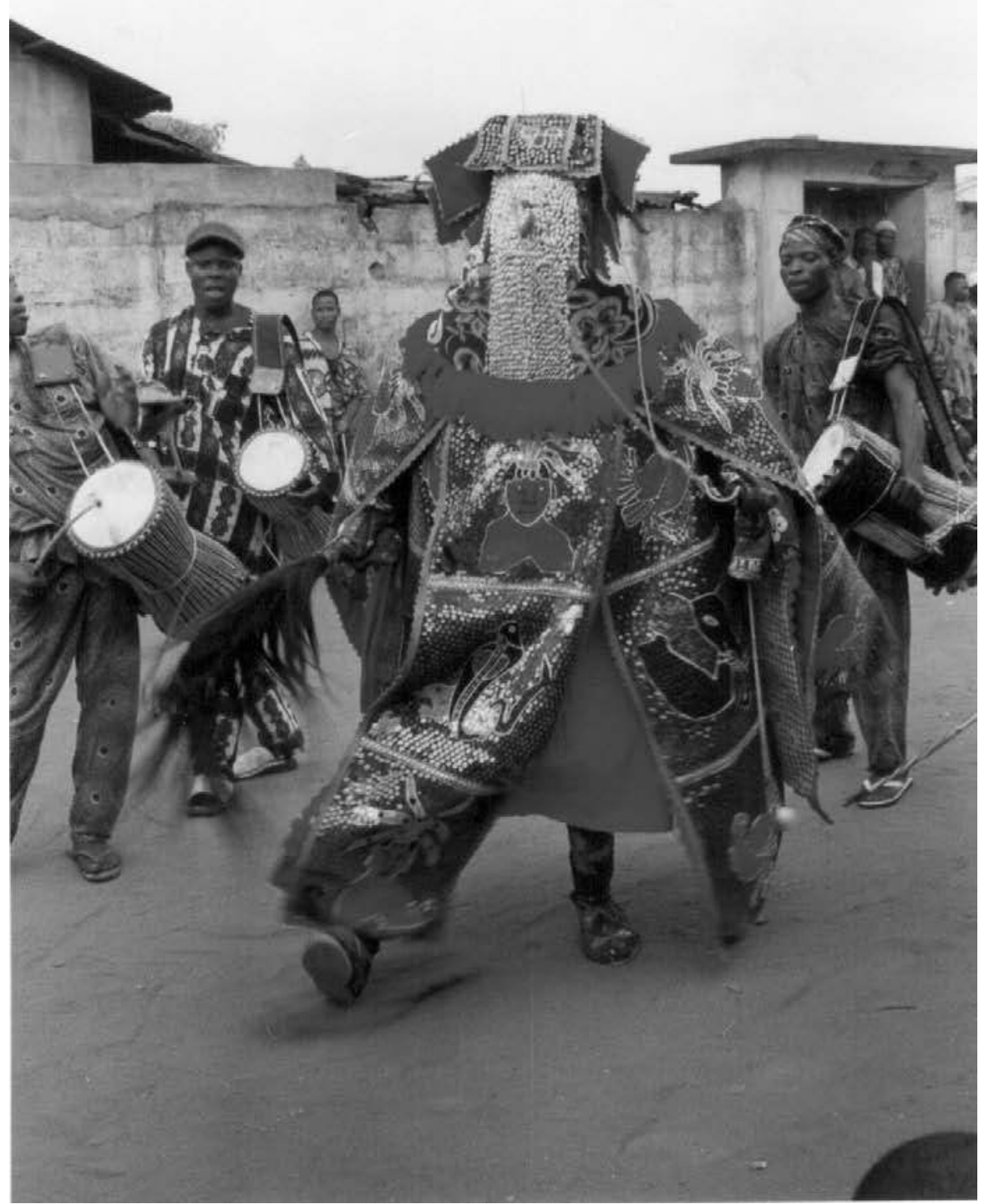

Fig. 6 Un egun s'élance au son des tambours gangan lors d'une sortie de masques, Ouidah, décembre 2003. Photo J. Noret. 
c'était réel ", entend-on souvent en substance). Et il n'est pas rare d'entendre des informateurs considérer que la forme prise par le culte dans les deux dernières décennies (caractérisées, en gros, par la multiplication des lignages détenteurs de masques et les occasions de sortie de ceux-ci) est pour ainsi dire galvaudée, les initiés ne s'avérant plus capables de conserver au culte son secret et sa puissance. Toutefois, cette forme de nostalgie, qui porte plus largement sur bon nombre de cultes considérés comme " traditionnels ", et à laquelle on se laisse surtout aller entre initiés (pour ne pas nuire au prestige du culte, qu'il s'agit évidemment de défendre aux yeux de ceux qui n'en sont pas), cohabite sans trop de difficultés avec le statut d'héritiers d'un culte attractif que bon nombre de Yoruba ont acquis avec l'émergence des egun dans la région d'Abomey.

Véritable " chevron ", c'est-à-dire, à l'inverse du stigmate, marqueur identitaire positif (voir Javeau 1997; Vienne 2004) désormais associé aux Yoruba, le culte egun a accompagné l'affirmation et la reconnaissance de populations autrefois stigmatisées, les posant en détentrices véritables d'un culte légitime. Et l'appropriation du culte par des lignages yoruba n'ayant vraisemblablement pas connu autrefois cette forme de culte aux ancêtres montre bien que les egun sont aujourd'hui devenus l'emblème d'une " tradition yoruba " supposée. Ainsi, à la fin des années 1980, un autel egun (mais aussi oro) fut installé également dans la concession lignagère Guèdègbe où, on l'a vu, on avait adopté jusquelà des rites de naissance et de mort fon, et où le fondateur du lignage avait, dans les premières décennies du $\mathrm{xx}^{\mathrm{e}}$ siècle, refusé que les premiers egun à sortir dans la ville se produisent dans sa maison ou dans ses alentours immédiats, montrant bien par là qu'il ne se reconnaissait pas vraiment dans ce culte. En fait, comme me le confiait en substance, et avec une certaine gêne, le sranon (l'un des adjoints du chef de lignage) de la maison en août 2005 (et à nouveau en 2007, lorsque je lui reposai la question), la maison Guèdègbe étant considérée comme le siège des cultes yoruba (Guèdègbe était le responsable des vodun yoruba installés à Abomey au XIX ${ }^{\text {e }}$ siècle, voir supra), ne pas avoir d'autel des cultes egun et oro commençait alors à poser un problème (en fait, un déficit de légitimité) vis-à-vis des autres lignages yoruba de la ville. Pour conserver une place de lignage prééminent dans le domaine des cultes yoruba, installer un tel autel devenait indispensable, et les autorités lignagères Guèdègbe décidèrent donc de revenir à la tradition d'origine supposée de la famille, « parce qu'on n'est plus au temps des rois ".
Se basant sur ce qu'elles pensaient être l'origine des parents de Guèdègbe et inférant sur ce qui s'était déjà passé dans bon nombre d'autres lignages, ces autorités décrétèrent donc que ce dernier était chogun choro (adepte à la fois d'egun et d'oro). Elles installèrent alors un nouvel autel ancestral associant les matérialisations des défunts des cultes egun et oro, une façon courante dans la région d'Abomey d'imbriquer ces deux formes historiquement distinctes de cultes rattachés, de très près ou de plus loin selon les régions du pays yoruba, aux ancêtres. On ne renonça pas à associer, lors de chaque décès, de petites matérialisations provisoires fon des défunts (kantonssin) aux artefacts formant le nouvel autel ancestral yoruba (car, toujours selon le sranon du lignage, " quand on est dans le pays de quelqu'un, il faut faire nécessairement ses cérémonies »), mais le lignage Guèdègbe commença vers 1990 à pratiquer des rites lignagers yoruba du type de ceux pratiqués dans d'autres lignages yoruba d'Abomey. Et en janvier 2004, après une célébration catholique organisée devant la concession lignagère, le cercueil du daa (titre fon des chefs de lignage) Guèdègbe fut ainsi transporté vers son lieu d'inhumation par des egun, accompagnés des voix vibrantes des oro $^{12}$.

\section{Des mémoires contrastées et contestées}

Dans un tel contexte d'identification du culte egun avec le monde yoruba, on comprend comment des masques egun furent associés à la Porte du Non-Retour lors de son édification sur la plage de Ouidah au moment du festival Ouidah 92 et de la " grande ritualisation dans l'arène politique » béninoise de la mémoire de la traite transatlantique (Bako-Arifari 2000 : 229). Les deux statues représentant des masques, placées de part et d'au-

12. Observation aux funérailles de daa Guèdègbe, 31 janvier 2004. On voit ici que les questions de l'émiettement de la mémoire des origines (Bastide 1970 : 95-108) et de la production de synthèses religieuses originales dans les mondes sociaux victimes, de différentes façons, de la traite esclavagiste ne concernent évidemment pas les seules «Amériques noires 》. II peut dès lors être tentant de considérer l'affirmation du culte egun dans la région d'Abomey comme un cas supplémentaire d'«invention » d'une tradition. Mais lorsque la construction d'une tradition est aussi étroitement imbriquée à celle du remaniement des places dans une chaîne de filiation, la métaphore de l'invention, largement répandue aujourd'hui, n'est peut-être pas la plus suggestive pour éclairer le phénomène. À tout prendre, en effet, le caractère limité ou la précarité des ressources dont disposent ici les acteurs (ancrés dans les conditions historiques difficiles de transmission d'une mémoire familiale bien souvent «trouée »] et les diverses contraintes ayant pesé sur le processus inclineraient à lui préférer la métaphore, peut-être plus suggestive en l'espèce, du bricolage (Lévi-Strauss 1990 [1962] : 30-36 ; Bastide 1970; Mary 2000]. 


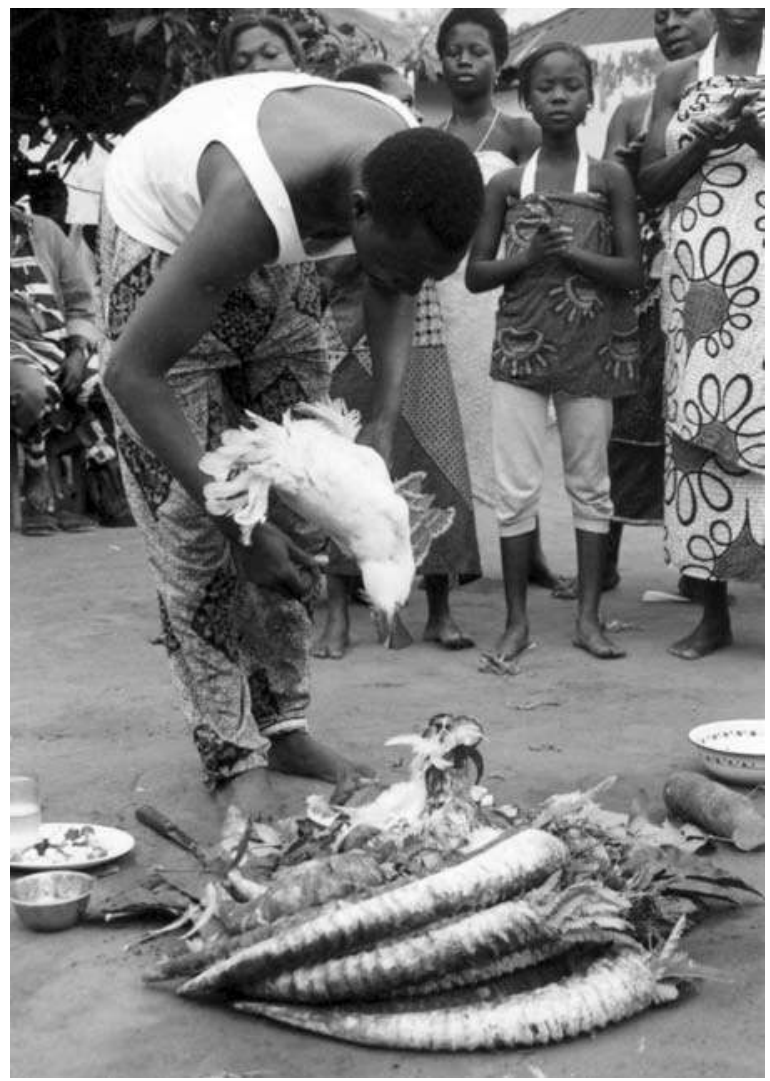

Fig. 7 Sacrifices annuels aux orichas (divinités yoruba) dont la famille à la charge dans la concession Guèdègbe, Abomey, août 2005. Photo J. Noret.

tre de la porte, étaient ainsi censées représenter «les esprits des esclaves ". Un tel choix n'allait pas de soi, dans la mesure où tous les Yoruba du Bénin méridional (ou même de l'ancien royaume du Dahomey) ne furent pas esclaves, et où tous les esclaves du Dahomey n'étaient pas yoruba (tous les "Yoruba " n'étant pas en outre concernés par le culte de la même manière). Mais une telle emblématisation des egun dans le cadre d'un projet patrimonial majeur comme celui de la Porte du NonRetour ne fit évidemment que renforcer l'association Yoruba-esclaves-egun.

Cette mémoire officielle des élites patrimonialisatrices trouva d'ailleurs un relais à Abomey avec le moulage par Yves Kpède, sculpteur reconnu dans le Bénin méridional et associé au festival Ouidah 92, d'une statue d'egun analogue à celles de la Porte du Non-Retour, le long de la voie que les esclaves empruntaient, juste après leur sortie d'Abomey, pour se rendre à Ouidah, voie que cherchent aujourd'hui à voir reconnue comme patrimoine mondial les promoteurs de la partie béninoise du projet international de la route de l'Esclave. Ainsi, comme me le racontait Kpède en août 2007, " après Ouidah 92, je me suis dit qu'il fallait quand même faire quelque chose autour de cette route et de cette région qui a quand même une histoire. On a voulu donner à cette voie son importance ». Comme à la Porte du NonRetour, Kpède choisit de mouler un egun, la « représentation la plus représentative " des ancêtres chez les "Yoruba ". Il ajouta sur le sommet du masque un cauri, symbole des richesses que les rois d'Abomey tirèrent de l'esclavage, et mit un miroir dans l'une des mains de l'egun pour évoquer les "pacotilles " reçues par les rois en échange des esclaves.

On le voit, la mémoire officielle de la traite, en plus d'encourager le recouvrement entre Yoruba et culte egun, associe allègrement ce dernier aux anciens esclaves. On peut même suggérer qu'en reproduisant des egun pour évoquer avant tout la traite transatlantique, cet artiste évoque aussi malgré lui l'esclavage local, puisque c'est bien évidemment par les egun du Bénin méridional actuel (et donc par les descendants des esclaves dont la route ne s'est pas poursuivie au-delà d'Abomey ou de Ouidah) qu'est inspiré un artiste comme Kpède. Simultanément, ces dynamiques patrimonialisatrices à l'égard des cultes "traditionnels " actives au Bénin dans les années 1990 (voir Bako-Arifari 2000; Tall 2005) ont évidemment bénéficié au culte egun, les initiés trouvant là des points d'appui bienvenus pour relativiser les diabolisations, chrétiennes en particulier, dont leur culte fait l'objet.

Devenus des symboles majeurs de l'identité yoruba, les egun sont donc susceptibles d'être mobilisés aussi par les commémorations de la traite transatlantique. À Abomey, ce sont toutefois les articulations entre mémoires de l'esclavage local et investissement dans le culte egun qui sont les plus significatives. D'une part, l'association entre egun et esclaves fait depuis longtemps partie du regard porté par les Fon sur le culte (voir l'anecdote rapportée par Melville Herskovits évoquée ci-dessus) et, dans bien des lignages fon, on sait (et on déclare avec plus ou moins de précautions langagières) que c'est à cause de la présence autrefois dans le lignage d'esclaves yoruba qu'on a aujourd'hui acquis le culte (ainsi, bien souvent, que le culte oro). Mais d'autre part, sans que cette première perspective soit contestée, celui-ci n'est, dans les familles ou les lignages yoruba d'Abomey qui se sentent aujourd'hui concernés par le culte des egun, pas tant associé au passé de déportés ou d'esclaves des pères qu'aux origines familiales supposées, et il permet plutôt de renouer avec un passé lignager antérieur à l'expérience de l'assujettissement, ce qui pose de facto celle-ci comme un moment bien circonscrit dans une histoire plus longue. 
Tout d'abord donc, si le culte des egun a bien acquis aujourd'hui une certaine légitimité à Abomey, son association avec les esclaves est pour ainsi dire inévitable dans les lignages fon qui ont acquis le culte, bien souvent en faisant valoir le souhait ou la nécessité (révélée par des consultations oraculaires) de faire désormais mémoire de la place qu'ont occupée en leur sein d'anciens esclaves, et en particulier d'anciennes esclaves, prises autrefois comme épouses par les hommes du lignage. Le terme de mèhiho (littéralement « personne achetée ") est toutefois largement considéré aujourd'hui comme une insulte et, en temps normal, l'usage est plutôt d'euphémiser les anciennes relations de domination, en mentionnant par exemple qu'il y avait des Yoruba dans la famille ou que « notre grand-mère était yoruba ", sans préciser d'emblée la condition servile des individus. Mais lorsque, pour telle ou telle raison, il faut évoquer une hiérarchie, le registre de l'esclavage est susceptible de remonter rapidement à la surface. Dans certains milieux, la stigmatisation du culte egun comme culte d'esclaves n'a pas complètement disparu : pour dire les choses rapidement, un certain nombre de membres de l'élite traditionaliste aboméenne conservent encore aujourd'hui une nostalgie certaine de l'époque où leurs ancêtres étaient les véritables maîtres du pays, dignitaires ou auxiliaires importants à la cour royale. Dans les dernières années, j'ai ainsi plusieurs fois entendu désapprouver un dignitaire important du culte egun à Abomey directement issu d'un grand prince : il lui était reproché de délaisser les traditions de son propre lignage paternel pour s'intéresser à un culte qui ne le concernait qu'en ligne maternelle. Ou encore, j'ai parfois entendu parler des cérémonies lignagères yoruba comme de « choses des esclaves », bien moins dignes de respect que l'héritage des rois. Mais de tels classements ont aujourd'hui un caractère ponctuel, l'ancienne division entre maitres et esclaves ayant à bien des égards perdu en pertinence au quotidien dans la société contemporaine, même si elle reste mobilisable en particulier en cas de conflit inter- ou intralignager ${ }^{13}$.

La position jadis occupée par un lignage dans le système esclavagiste n'est pas, je l'ai dit, le seul élément qui soutient aujourd'hui la formation d'une mémoire de l'esclavage. Le niveau d'éducation scolaire et la socialisation religieuse, par exemple, interviennent aussi de façon manifeste dans le façonnage de ces mémoires. Ainsi, lorsque j'évoquai avec daa Houinato, héritier d'une lignée d'alliés proches du roi Guezo (1818-1858) responsable sous son règne au moins de la garde des captifs de guerre fraîchement arrivés à Abomey, l'histoire de son lignage
- l'une des premières choses qu'il m'expliqua, faisant allusion aux anciens esclaves et captifs morts dans sa maison sans que leur soient données des funérailles convenables -, celui-ci me dit qu'il y avait « des âmes qui sont là autour de nous », et qu'il fallait " quand même faire quelque chose pour se souvenir de ces âmes-là ", "mortes dans de grandes souffrances, avec violence »: c'était là sa « compréhension religieuse » de la situation. Haut fonctionnaire de l'État béninois et catholique, il avait dès lors l'intention d'organiser prochainement des cérémonies egun et oro, mais d'en déléguer en quelque sorte l'exécution à d'anciennes familles dépendantes du quartier sans installer d'autel yoruba dans sa propre concession lignagère parce qu'il s'agissait de « choses qu'il faut entretenir dans la durée » et qu'il était plus facile, et plus prudent, de "s'appuyer sur des gens qui ont l'habitude de le faire » et dont c'est " la chose $\mathrm{e}^{14}$ ».

À la tête d'un lignage important revendiquant un certain nombre de lignages dépendants, daa Houinato soulignait aussi les ambiguïtés que charrie encore aujourd'hui la question de l'esclavage local dans la gestion d'une grande collectivité comme la sienne. «Prêt pour l'histoire ", selon ses propres termes, il évoquait de façon bien plus directe que d'autres informateurs les questions essentielles qui se posent toujours aujourd'hui dans bien des lignages fon autour de la place qu'y occupent des lignées familiales issues d'esclaves ou de dépendants : «Il y a des gens qui sont même devenus des parents. On ne peut même plus en parler aujourd'hui. Il y en a qui signent Houinato comme moi. Mais on le sait toujours, car ces gens n'ont pas droit au trône [lignager]. " Dans certains lignages toutefois, à Abomey ou à

13. La problématique du stigmate à travers laquelle je lis ici la trajectoire du culte egun dans la région d'Abomey s'écarte assez de la problématique du trauma dans laquelle Nicolas Argenti a récemment inscrit l'essentiel de son interprétation des performances des masques du palais de la chefferie d'Oku dans les Grassfields du Cameroun contemporain (Argenti 2006), stigmate et trauma ne pouvant évidemment pas être rabattus l'un sur l'autre. Je ne dispose pas ici de suffisamment d'espace pour discuter vraiment du schéma interprétatif d'Argenti (même si le statut de mémoire traumatique qu'il confère aux traces vraisemblablement laissées par le système esclavagiste sur la société de masques qu'il évoque ne me paraît pas aller de soi]. II me semble toutefois important de noter que (1) les modes dominants de production des esclaves dans la région d'Abomey (où ceuxci provenaient largement de razzias opérées par l'armée du Dahomey dans des régions voisines) et dans les Grassfields (où ceux-ci provenaient plutôt de sentences prononcées par les détenteurs du pouvoir à l'encontre de membres de la société), et (2) les places différentes occupées par le royaume du Dahomey et par la chefferie d'Oku dans l'économie de la traite permettent certainement de rendre compte pour partie des formes distinctes prises par les mémoires de l'esclavage dans l'un et l'autre cas.

14. Entretien avec daa Houinato Adimalon, août 2007. 
d'être un revenant, de lui pratiquer les coutumes de son origine ancestrale, d'Oyo, de lui pratiquer les coutumes qui sont les siennes, d'Oyo. » Issue d'un homme « déporté » des alentours de Dassa-Zoumè (à une centaine de kilomètres au nord d'Abomey) par une razzia des troupes de Béhanzin en 1889, mais qui aurait été, toujours selon Damien Agliti, un commerçant yoruba originaire d'Oke Odan, en pays yoruba, la famille Agliti a probablement bien peu à voir, historiquement, avec la région d'Oyo, ville située à quelques centaines de kilomètres au nordest des expéditions les plus aventureuses des troupes du Dahomey (lesquelles ne dépassèrent jamais Abeokuta). Mais, comme en bien d'autres cas, la revendication d'une origine à Oyo vient probablement du prestige du nom et du fait que l'un des noms les plus régulièrement donnés aux Yoruba en fon est Ayonu (littéralement "gens d'Oyo "). Le culte egun joue alors ici le rôle de médiateur privilégié avec cette origine familiale supposée, dont il atteste l'authenticité.

Mais le passage par l'expérience aliénante de l'esclavage n'est pas nécessaire pour qu'on entreprenne une telle réévaluation des origines. Après tout, certains captifs amenés au Dahomey eurent, semble-t-il, assez rapidement accès à des formes d'affranchissement. Ainsi le lignage Guèdègbe est-il bien issu d'une famille amenée à Abomey depuis Savè, mais son fondateur, "Guèdègbe $I^{\mathrm{er}}$ ", comme on dit aujourd'hui, s'est trouvé libre avant la fin de sa vie en raison de ses compétences divinatoires, reconnues à la cour royale. L'appropriation récente dans ce lignage de cérémonies lignagères yoruba chogun choro, évoquée plus haut, s'y justifie toutefois par le même genre de regard rétrospectif. Le sranon de la maison m'expliquait ainsi en août 2007 : « Nous, nous sommes d'Oyo, et nous allons “aller à Agon" [nom d'une cérémonie essentielle des rites funéraires fon tels qu'ils étaient pratiqués dans le lignage jusqu'à il y a une vingtaine d'années] ? Nous sommes [serions] en train de nous perdre. " Pratiqués par les Guèdègbe pendant plusieurs décennies, les rites fon de naissance et de mort sont ici désormais considérés comme les témoins d'une aliénation passée.

Bien entendu, c'est fatalement dans le présent qu'on se rapporte au passé (Lenclud 1987), et les réaménagements de la filiation qui accompagnent les installations d'autels egun sont désormais ancrés dans un contexte où l'identité de détenteur du culte fait l'objet d'une certaine reconnaissance sociale. Taisant toutefois le plus souvent cette lutte pour la reconnaissance, les récits produits par les informateurs sur leur propre lignage justifient plutôt largement l'appropriation des cultes egun et oro par la fidélité aux origines. Or, si c'est donc surtout par sa filiation qu'on justifie son investissement dans le culte, il en va différemment du regard porté sur l'acquisition de ces cultes par les autres lignages. Bon nombre d'informateurs sont ainsi prompts à remettre en cause l'authenticité des liens historiques qui lient aux cultes en question telle ou telle famille qui «se dit propriétaire de la chose ". De telles dénonciations sont l'un des registres majeurs des querelles de légitimité qui opposent lignages et quartiers autour de leur bon droit à pratiquer ce qui est, ou n'est pas, «pour leurs pères ». Ressort de l'honorabilité de la filiation et de l'identité lignagère dans bon nombre de familles, le culte egun est aussi, fatalement, au cœur de conflits et de tensions entre mémoires de l'origine au sein même de la « communauté " yoruba.

La complexité sociale, identitaire, et donc mémorielle, léguée par l'esclavage local dans le Bénin méridional est manifeste. À Abomey, le culte des egun est ainsi, depuis plusieurs décennies maintenant, au cœur de compromis et de dynamiques identitaires qui ne peuvent faire abstraction du système esclavagiste fon. Investie par un nombre croissant de lignages yoruba au cours du $\mathrm{xx}^{\mathrm{e}}$ siècle, cette forme de culte des ancêtres a été dans ce milieu un vecteur essentiel de la restauration d'une mémoire honorable des origines, capable de faire contrepoids au stigmate de l'esclavage (ou de l'assujettissement) par la convocation d'une identité antérieure prestigieuse, au point que l'investissement dans le culte est devenu aujourd'hui un support privilégié de l'affirmation d'une filiation yoruba. Pour autant, le culte des egun n'a évidemment pas fait oublier le passé esclavagiste de la région, la logique de l'acquisition du culte dans les lignages fon passant bien souvent par le souhait de faire une place à la tradition supposée d'ancien(ne)s esclaves. En outre, depuis les années 1990, les initiatives politiques de patrimonialisation ont ajouté une nouvelle couche à l'histoire du culte egun dans la région d'Abomey. Désormais symbole incontournable sur la scène nationale d'une certaine yorubaïté, le culte a aussi trouvé dans l'intérêt patrimonial une nouvelle forme de légitimité culturelle qui relativise la diabolisation croissante dont, à l'instar des autres cultes assimilés à l'univers du vodun, il fait l'objet dans le chef des élites chrétiennes et musulmanes et de leurs audiences.

Enfin, il a beaucoup été question dans les pages qui précèdent de sentiment d'authenticité, de fidélité à soi et de persistance. En effet, le culte des egun est dans bien des lignages yoruba le gage ou le signe tangible d'une forme de continuité lignagère par-delà l'expérience de 


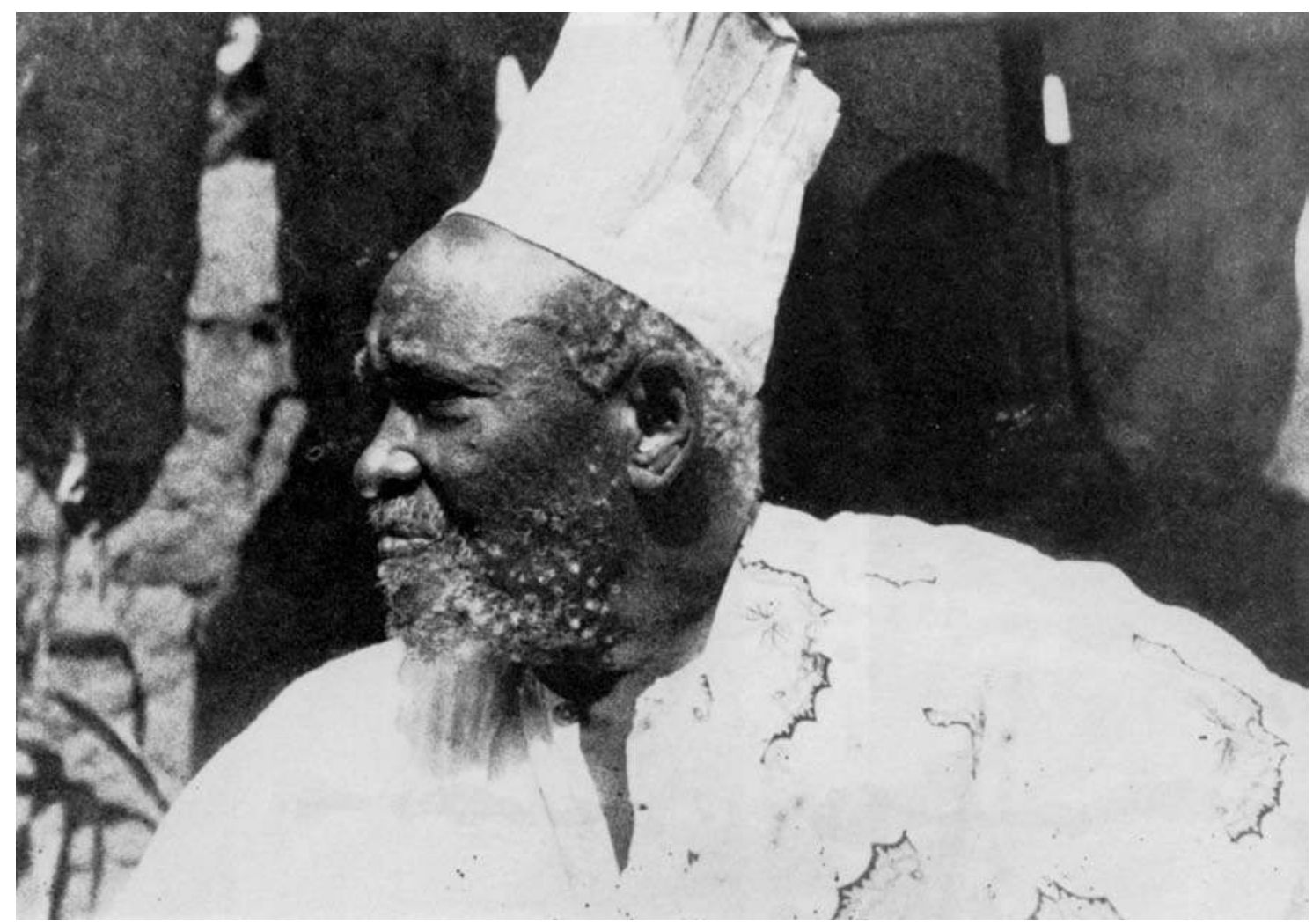

Fig. 9 Bernard Maupoil, le devin Guèdègbe, interlocuteur privilégié de Bernard Maupoil, Abomey, 1935, DR.

l'esclavage. Dans bon nombre de lignages fon, la présence du culte atteste d'autres aspects du passé lignager, mais il renvoie toujours à la pérennité du lignage, qui fait aujourd'hui une place à ses dépendants ou à ses épouses d'autrefois. Or, un tel tableau, certes brossé à gros traits, laisse dans l'ombre une autre forme de relation mémorielle qui se greffe fréquemment aussi sur le culte des egun aujourd'hui. En effet, les rapports au passé peuvent ne pas être faits d'un bloc (relever seulement du sentiment d'authenticité ou au contraire de la nostalgie, par exemple) et, je l'ai évoqué trop rapidement, la revendication d'une forme de continuité se conjugue chez bien des adeptes avec un sentiment de perte et une certaine nostalgie à l'égard de ce que le culte est considéré avoir été à ses débuts : les egun d'aujourd'hui ne sont plus ceux d'hier. Selon les contextes et les discussions, c'est l'un ou l'autre de ces registres, l'une ou l'autre de ces façons de penser, qui l'emporte. Le sentiment de perte toutefois ne sape pas vraiment la fierté qui découle de la continuité lignagère supposée mise en scène par le culte. Pris entre la nostalgie des forces magiques dont ils considèrent que les générations antérieures étaient les pleines dépositaires et la conviction qu'ils perpétuent malgré tout leur héritage, bon nombre d'adeptes du culte des egun dans la région d'Abomey mobilisent ainsi régulièrement deux façons de voir, deux regards sur le passé dont on peut considérer qu'ils sont tous deux intériorisés (en fait, nourris par les discours plus larges sur l'authenticité et sur la perte qui circulent dans la postcolonie béninoise) et qu'ils constituent un bon exemple d'hétérogénéité interne des acteurs (voir Lahire 1998, 2002). Ou, pour le dire autrement, sentiment d'authenticité et de continuité d'une part, de nostalgie et de perte d'autre part cohabitent chez bien des initiés comme deux parties d'eux-mêmes, sans donner lieu trop souvent à des arbitrages entre façons de voir. C'est là évidemment une ambiguïté supplémentaire des recompositions identitaires qui s'articulent au culte des egun qu'il eût été difficile de ne pas rappeler.

mots clés / keywords : Yoruba // Yoruba . Abomey // Abomey - tradition // tradition • authenticité // authenticity • esclavage // slavery • mémoire // memory.

Chargé de recherche au FNRS-Belgique jnoret@ulb.ac.be 


\section{Bibliographie}

\section{ANIGNikin, Sylvain}

2001 « Histoire des populations mahi. À propos de la controverse sur l'ethnonyme et le toponyme "Mahi" », Cahiers d'études africaines 162 : 243-266.

\section{Appadurai, Arjun}

1981 « The Past as a Scarce Resource », Man (nouvelle série) 16[2] : 201-219.

\section{ARgenti, Nicolas}

2006 « Remembering the Future. Slavery, Youth and Masking in the Cameroon Grassfields », Social Anthropology 14[1] : 49-69.

\section{Argenti, Nicolas et Röschenthaler,} Ute

2006 «Introduction. Between Cameroon and Cuba. Youth, Slave Trades and Translocal Memoryscapes », Social Anthropology 14[1] : 33-47.

\section{Augé, Marc}

1982 Génie du paganisme. Paris, Gallimard.

\section{BAKO-ARIFARI, Nassirou}

2000 «La mémoire de la traite négrière dans le débat politique au Bénin dans les années 1990 », Journal des africanistes $70(1-2)$ : 221-231.

\section{BAstide, Roger}

1970 «Mémoire collective et sociologie du bricolage », L'Année sociologique (troisième série] 21 : 65-108.

\section{BAy, Edna}

1998 Wives of the Leopard. Gender, Politics, and Culture in the Kingdom of Dahomey. Charlottesville et Londres, University of Virginia Press.

2008 Asen, Ancestors, and Vodun. Tracing Change in African Art. Champaign, University of Illinois Press.

\section{BerLiner, David}

2005 « The Abuses of Memory: Reflections on the Memory Boom in Anthropology 》, Anthropological Quarterly 78(1) : 197-211.

\section{ClifFord, James}

1986 «Introduction: Partial Truths », in James Clifford et George Marcus (éd.), Writing Culture. The Poetics and Politics of Ethnography. Berkeley, University of California Press : 1-26.

Herskovits, Melville

1938a Dahomey. An Ancient West African Kingdom, volume I. New York, Augustin Publisher.

1938b Dahomey. An Ancient West African Kingdom, volume II. New York, Augustin Publisher.

\section{JAVEAU, Claude}

1997 Leçons de sociologie. Paris, Armand Colin.

LAHIRE, Bernard

1998 L'Homme pluriel. Les ressorts de l'action. Paris, Nathan.

2002 Portraits sociologiques. Dispositions et variations individuelles. Paris, Nathan.

\section{LAMBEK, Michael et AnTze, Paul}

1996 «Introduction: Forecasting Memory », in Michael Lambek et Paul Antze [éd.], Tense Past. Cultural Essays in Trauma and Memory. Londres, Routledge : xi-xxxviii.

\section{LAW, Robin}

2004 Ouidah. The Social History of an African Slaving "Port". Athens et Oxford, Ohio University Press et James Currey.

\section{Le HÉRIssé, Auguste}

1911 L'Ancien Royaume du Dahomey. Mœurs, religion, histoire. Paris, Émile Larose.

\section{LENCLUD, Gérard}

1987 « La tradition n'est plus ce qu'elle était. Sur les notions de "tradition" et de "société traditionnelle" en ethnologie », Terrain 9 : 110-123.

\section{LÉVI-STRAUSS, Claude}

1990 [1962] La Pensée sauvage. Paris, Pocket.

MARY, André

2000 Le Bricolage africain des héros chrétiens. Paris, Cerf.
2003 «L'invention chrétienne de l'identité yoruba. Les racines missionnaires d'une nation africaine 》, Archives de sciences sociales des religions 124 : 49-61.

\section{MAUPOIL, Bernard}

1943 La Géomancie à l'ancienne côte des Esclaves. Paris, Institut d'ethnologie.

\section{Noret, Joël}

2004 « De la conversion au basculement de la place des morts. Les défunts, la personne et la famille dans les milieux pentecôtistes du Sud-Bénin », Politique africaine 93 : 143-155.

2006 Autour de «ceux qui n'existent plus 》. Deuil, funérailles et place des défunts au Sud-Bénin. Thèse de doctorat, Bruxelles et Paris, Université libre de Bruxelles et École des hautes études en sciences sociales.

\section{PeEL, John David Y.}

2000 Religious Encounter and the Making of the Yoruba. Bloomington et Indianapolis, Indiana University Press.

\section{Preston Blier, Suzanne}

1989 «Field Days. Melville J. Herskovits in Dahomey », History in Africa 16 : 1-22.

\section{SHAW, Rosalind}

2002 Memories of the Slave Trade. Ritual and the Historical Imagination in Sierra Leone. Chicago, University of Chicago Press.

TALL, Kadya

2005 «Stratégies locales et relations internationales des chefs de culte au SudBénin », in Laurent Fourchard, André Mary et René Otayek (éd.), Entreprises religieuses transnationales en Afrique de l'Ouest : 267-294. Ibadan-Paris, IFRA-Karthala.

VIEnNE, Philippe

2004 « Au-delà du stigmate : la stigmatisation comme outil conceptuel critique des interactions et des jugements scolaires », Education et Sociétés 13 : $177-192$. 


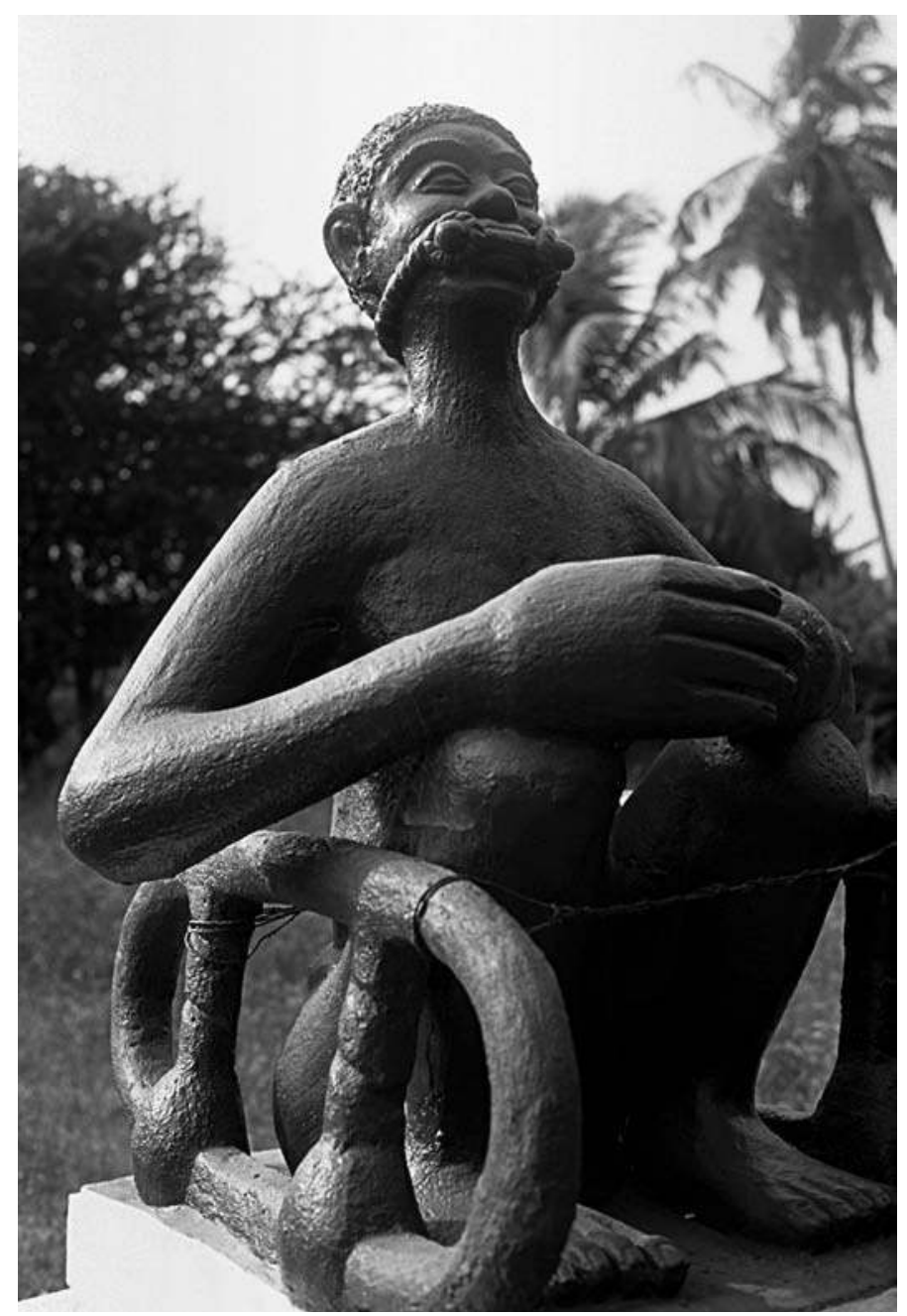

Fig. 10 Statue de Cyprien Toukoudagba représentant un esclave ligoté sur le site dit "Zomaï », La Route de l'Esclave, Ouidah, 2004. Photo M. Guran.

\section{Résumé / Abstract}

Joël Noret, Mémoire de l'esclavage et capital religieux. Les pérégrinations du culte egun dans la région d'Abomey. - Cet article porte sur les mémoires de l'esclavage dans la région d'Abomey, au cœur du pays fon et du royaume précolonial du Dahomey, telles qu'elles se donnent à voir à travers le culte egun, une forme yoruba de culte aux ancêtres. En effet, dans les débats qui ont entouré l'apparition puis le développement de ce culte, porté à ses débuts surtout par des Yoruba d'origine servile, on peut repérer des persistances et des glissements dans la place faite à l'esclavage dans la société aboméenne. On y lit simultanément l'émergence, dans les lignages se réclamant d'une origine yoruba, de tentatives d'affranchissement du stigmate de l'esclavage et d'une revendication d'authenticité yoruba. L'investissement dans le culte egun, d'abord toléré puis approprié par les Fon, est au cœur de ces processus.
Joël Noret, Memory of slavery and religious capital. The peregrinations of the Egun cult in the Abomey region. - This article concerns memories of slavery in the Abomey region, in the heart of Fon country and of the pre-colonial kingdom of Dahomey, such as they are revealed through the Egun cult, a Yoruba form of ancestor cult. In the debates which have surrounded the apparition and then the development of this cult, which began principally among Yoruba with slave origins, there are noticeable continuities and slippages in the place made for slavery in Abomey society. One discerns simultaneously the emergence, in the lineages claiming Yoruba origins, of attempts at liberation from the stigma of slavery and at a claim of Yoruba authenticity. Involvement in the Egun cult, at first tolerated and later appropriated by the Fon, is at the centre of this process. 